\title{
Liothyronine I-125
}

National Cancer Institute

\section{Source}

National Cancer Institute. Liothyronine 1-125. NCI Thesaurus. Code C80096.

A radioconjug ate of synthetic active thyroid hormone, liothyronine (T3), labeled with Iodine 125. Liothyronine involves many important metabolic functions and is essential to the proper development and differentiation of all cells. I-125 liothyronine may be used in radiotherapy in thyroid cancers. 